\title{
DESEMPENHO DE CORDEIROS MACHOS INTEIROS, MACHOS CASTRADOS E FÊMEAS, ALIMENTADOS EM CONFINAMENTO ${ }^{1}$
}

\author{
PERFORMANCE OF WHOLE MALES LAMBS, CASTRATED MALES \\ LAMBS AND FEMALES LAMBS, FEED IN CONFINEMENT
}

\author{
Sérgio Carvalho ${ }^{2}$ Cleber Cassol Pires ${ }^{3}$ João Ronaldo Ramos Peres ${ }^{4}$ \\ Carla Zeppenfeld $^{4}$ Alexandre Weiss ${ }^{4}$
}

\section{RESUMO}

O estudo foi realizado com o objetivo de avaliar o efeito do sexo sobre o ganho de peso, consumo de matéria seca e conversão alimentar de cordeiros confinados e abatidos aos 100 dias de idade. Foram utilizados 18 cordeiros $(6$ machos não castrados, 6 machos castrados e 6 fêmeas), filhos de carneiro Texel com ovelhas cruzas (Texel e Ideal). Os animais (ovelhas + cordeiros) foram confinados em baias individuais 24 horas após o parto até o desmame, que ocorreu aos 50 dias. Após, os cordeiros permaneceram sozinhos até o abate aos 100 dias de idade. $O$ alimento utilizado foi silagem de milho + concentrado (milho + farelo de soja + minerais), na proporção 58:42 na matéria seca. Os valores encontrados para ganho de peso, consumo de matéria seca (kg/animal/dia, \%PV e g/UTM) e conversão alimentar não diferiram $(P \geq 0,05)$ entre os sexos dos cordeiros. Os resultados obtidos mostram que cordeiros machos não castrados, castrados e fêmeas apresentam desempenho semelhante até os 100 dias de idade.

Palavras-chave: confinamento, conversão alimentar, ganho de peso, ovinos, sexo.

\section{SUMMARY}

The study was carried with the objective to evaluate the effect of the sex upon the weight gain, dry matter intake and food conversion of feedlot lambs and slaughtered at one hundred days of age. Eighteen lambs (six whole males, six castrated males and six females) descendents of a Texel male with (Texel $x$ Ideal) female sheeps, were used. The animals (sheeps + lambs) were confined in individuals bails 24 hours after delivery until the weaning at the 50 days. After, they remained along until the

\begin{abstract}
slaughter at the age of 100 days. The food used was corn silage + concentrated (corn + soybean meal + minerals), in the proportion 58:42 in dry matter. The values obtained for weight gain, dry matter intake (kg/animal/day), \% live weight and $\mathrm{g} / \mathrm{metabolic}$ size and food conversion, did not differ $(P \geq 0,05)$ among the sex of the lambs. The results obtained showed that whole males lambs, castrated males lambs and females lambs, present performance similar at the age of 100 days.
\end{abstract}

Key words: confinement, food conversion, sex, sheeps, weight gain.

\section{INTRODUÇÃO}

Os sistemas de produção de ruminantes adotados, mais notadamente os de ovinos, são predominantemente extensivos. Entretanto, alguns países como a Austrália, têm utilizado também sistemas intensivos sofisticados, sobretudo no que se refere ao confinamento de cordeiros para abate (SIQUEIRA et al., 1993).

No Rio Grande do Sul, tradicionalmente, a base da alimentação dos ovinos é a pastagem natural, sendo os animais criados de forma extensiva, o que torna difícil a obtenção de bons índices produtivos, bem como a produção de animais jovens em condições de serem abatidos. A utilização de pastagens cultivadas ou o fornecimento de suplementação alimentar são formas de suprir os animais durante os

\footnotetext{
${ }^{1}$ Parte da Dissertação do primeiro autor apresentada à UFSM para obtenção do título de 'Magister Science'.

${ }^{2}$ Zootecnista MSc. Produção Animal - UFSM - Depto. de Zootecnia - 97105-900 Santa Maria, RS.

${ }^{3}$ Professor Titular do Departamento de Zootecnia - UFSM - Depto. de Zootecnia - 97105-900 Santa Maria, RS. cpires@creta.ccr.ufsm.br Autor para correspondência.

${ }^{4}$ Aluno de graduação em Zootecnia - UFSM. 
períodos de carência. Esta última prática de alimentação vem crescendo nos últimos anos, podendo ser realizada tanto na forma de suplementação a campo, como no sistema de confinamento.

Para MOREIRA (1997), partir para o confinamento de ovinos é uma solução prática, porque traz como benefícios a diminuição da mortalidade, do índice de endo e ecto parasitas e da mão de obra, além de melhorar a eficiência e a produtividade do criatório. CASTELLÁ (1997) cita que o confinamento também proporciona uma maior facilidade de manejo do rebanho ovino, uma vez que não é necessário observarem-se os animais nos campos, através de recorridas das invernadas.

SIQUEIRA et al. (1993), realizando um estudo comparativo na recria de cordeiros em confinamento ou pastagem, observaram que o ganho de peso diário e o peso vivo final foram superiores nos cordeiros confinados. Outro fator importante, constatado neste estudo, foi que as infecções endoparasitárias (helmintíases) foram maiores no grupo recriado a campo, fato que explica o pior desempenho do mesmo, já que a condição nutricional da pastagem era boa.

Aliado ao confinamento, o sexo é outro fator importante que se dispõe para a obtenção de bons ganhos de peso. AZZARINI (1979) verificou que o sexo afeta a velocidade de crescimento e a deposição dos distintos tecidos do corpo dos animais, sendo a velocidade de crescimento maior nos machos não castrados do que nos castrados (9\%) e maior nestes do que nas fêmeas $(5 \%)$. Já CROUSE et al. (1981) afirmam que a performance superior, obtida por machos inteiros, pode ser incrementada em níveis altos de alimentação.

O objetivo deste trabalho foi de avaliar o efeito do sexo sobre o ganho de peso, consumo de matéria seca e conversão alimentar de cordeiros confinados e abatidos aos 100 dias de idade.

\section{MATERIAL E MÉTODOS}

O experimento foi conduzido nas dependências do Setor de Ovinocultura do Departamento de Zootecnia da Universidade Federal de Santa Maria, no período de 25 de julho a 23 de novembro de 1996.

Foram utilizados 18 cordeiros oriundos do acasalamento de carneiro da raça Texel com ovelhas cruza (Texel e Ideal), sendo 6 machos não castrados (T1), 6 machos castrados (T2) e 6 fêmeas (T3). Os animais (cordeiros e respectivas mães), 24 horas após o parto foram confinados em baias individuais com piso ripado, a uma altura de 1 metro acima do solo com área de $4 \mathrm{~m}^{2}$ e providas de comedouros e bebedouros.
As ovelhas e cordeiros foram pesados individualmente 24 horas após o parto, aos 25 e 50 dias. Posteriormente, aos 75 e 100 dias apenas os cordeiros foram pesados. Foi observado jejum de sólidos para a pesagem final, não o sendo para as pesagens intermediárias. 6 machos foram castrados aos 7 dias de idade, com uso de anel de borracha. $\mathrm{O}$ descole não foi realizado em nenhum dos animais distribuídos nos tratamentos. Todos os cordeiros foram desmamados aos 50 dias de idade, quando a ovelha (mãe) era retirada do confinamento.

Foram utilizadas duas dietas experimentais constituídas por silagem de milho + concentrado, na proporção 58:42, na MS. Inicialmente, foi fornecida uma ração específica para atender às exigências nutricionais das ovelhas. $\mathrm{O}$ concentrado utilizado nesta dieta era constituído por milho desintegrado, farelo de soja, calcário calcítico e cloreto de sódio. Após o desmame (50 dias), o concentrado foi modificado no sentido de atender às exigências nutricionais dos cordeiros, sendo constituído por milho desintegrado, farelo de soja com $48 \%$ de proteína bruta (Protosan), calcário calcítico e cloreto de sódio. Ambas dietas utilizadas foram calculadas de acordo com o NRC (1985).

Os animais foram alimentados duas vezes ao dia, sendo os horários de alimentação às 8 e 17 horas. A quantidade oferecida foi ajustada em função da sobra observada diariamente. Esta foi controlada para que fosse $20 \%$ da quantidade oferecida no dia anterior, de modo a garantir o consumo voluntário dos animais. Registravam-se, diariamente, as quantidades de alimento fornecido individualmente aos animais. As sobras eram pesadas e amostradas diariamente, assim como o alimento oferecido, sendo acondicionados e congelados para posterior determinação de matéria seca.

Foi utilizado o delineamento experimental inteiramente casualizado, em um total de três tratamentos com 6 repetições cada. Foi realizada a análise de variância dos dados, para verificar o efeito do sexo sobre as características utilizadas, através do pacote estatístico SAS (1990).

\section{RESULTADOS E DISCUSSÃO}

Os valores médios para peso inicial, peso ao desmame, peso final e ganhos médios diários do nascimento ao desmame, do desmame aos 100 dias e do nascimento aos 100 dias, são apresentados na Tabela 1.

Observa-se que não houve diferenças significativas $(\mathrm{P} \geq 0,05)$ para nenhuma das variáveis estudadas. Em termos de valores numéricos, os resultados obtidos são concordantes com AZZARINI 


\begin{tabular}{|c|c|c|c|c|c|c|}
\hline TRATAMENTO & PI & PD & $\mathrm{PF}$ & GMDA & GMDB & GMD \\
\hline Macho Inteiro & 4,16 & 19,98 & 27,91 & 0,316 & 0,159 & 0,237 \\
\hline Macho Castrado & 4,58 & 20,22 & 27,28 & 0,313 & 0,141 & 0,227 \\
\hline Fêmea & 4,40 & 19,37 & 26,07 & 0,299 & 0,134 & 0,217 \\
\hline Média & 4,38 & 19,85 & 27,09 & 0,309 & 0,145 & 0,227 \\
\hline Erro Padrão & 0,28 & 0,59 & 1,33 & 0,01 & 0,02 & 0,01 \\
\hline $\mathrm{F}$ & 0,55 & 0,54 & 0,49 & 1,27 & 0,31 & 0,72 \\
\hline $\mathrm{P}>\mathrm{F}$ & 0,58 & 0,59 & 0,62 & 0,31 & 0,73 & 0,50 \\
\hline CV $(\%)$ & 15,82 & 7,35 & 12,11 & 6,35 & 38,25 & 13,26 \\
\hline
\end{tabular}

$(\mathrm{P} \geq 0,05)$. requerimentos das ovelhas, o que permitiu que estas mantivessem uma alta produção diária de leite. Sendo assim, a participação do leite na alimentação dos cordeiros no momento do desmame ainda era grande, trazendo como conseqüência, um baixo consumo do alimento fornecido (volumoso + concentrado) por parte do cordeiro, enquanto estava com a mãe. Com a retirada da ovelha do confinamento, a ausência do leite foi sentida pelos cordeiros, principalmente nos primeiros 25 dias pósdesmame (mesmo que o alimento fornecido tenha sido formulado para atender às exigências nutricionais dos cordeiros), quando houve um baixo consumo, uma vez que os cordeiros estavam se adaptando à nova fase de vida e ao alimento fornecido, pois passaram a depender exclusivamente desse.

A afirmativa acima é corroborada pelos (1979), FIGUEIRÓ \& BENAVIDES (1990), que afirmam que machos não castrado têm velocidade de crescimento maior que a dos castrados e estes maior que a das fêmeas. Concordam também com SENTS et al. (1982), os quais citam que, geralmente, machos não castrados crescem mais e com mais eficiência, bem como apresentam uma menor percentagem de gordura do que castrados e fêmeas.

Provavelmente, a idade muito jovem de abate tenha sido um fator limitante para que não houvesse diferenças acentuadas em relação ao desempenho dos animais. Possivelmente, em uma idade de abate mais avançada haveria maior ação do hormônio masculino (testosterona), proporcionando benefícios para os machos não castrados em relação aos castrados e às fêmeas. Conforme JACOBS et $\boldsymbol{a l}$. (1972), o desenvolvimento de características secundárias em machos não castrados é atribuída à produção de testoterona; esta aumenta a eficiência alimentar, bem como é uma promotora do crescimento muscular e esquelético, sendo que estes efeitos se acentuam após a puberdade. Trabalhando com uma idade mais avançada de abate, JENKINS $\boldsymbol{e t}$ al. (1988) observaram um GMD de $340 \mathrm{~g}$, 298g e $270 \mathrm{~g}$ para machos não castrados, castrados e fêmeas, submetidos as mesmas condições alimentares dos 83 até 167 dias de idade.

Avaliando o ganho de peso por período, verifica-se que houve uma drástica redução após o desmame $(0,309 \mathrm{~kg}$ para $0,145 \mathrm{~kg}$ de GMD em média). Esta redução pode ser atribuída à ausência de leite materno e ao baixo consumo de alimento pelos cordeiros, principalmente nos dias logo após o desmame.

No presente trabalho, na fase de lactação, utilizou-se um alto nível nutricional para atender aos resultados apresentados na Tabela 2 , onde se verifica que houve uma elevação na quantidade de alimento consumido pelos cordeiros, à medida que passou o tempo do desmame. Pode-se observar que o consumo médio diário de matéria seca, que foi de $0,47 \mathrm{~kg} /$ animal no primeiro período pós-desmame (50 a 75 dias), passou para $0,66 \mathrm{~kg} /$ animal no segundo período (75 a 100 dias), sendo este aumento de $40,4 \%$.

Os valores para consumo de matéria seca diário ( $\mathrm{kg} / \mathrm{animal} / \mathrm{dia})$, para $100 \mathrm{~kg}$ de peso vivo $(\%$ PV) e em função do tamanho metabólico (g/UTM) e a conversão alimentar, de ovelha + cordeiro do nascimento aos 50 dias de idade estão apresentados na Tabela 3 e para os cordeiros dos 50 aos 100 dias, estão na Tabela 4.

Na Tabela 3, o consumo de matéria seca foi calculado em função do peso médio do conjunto ovelha + cordeiro e a conversão alimentar foi calculada em função da quantidade de matéria seca consumida por ovelha + cordeiro, em kg, para que houvesse o ganho de $1 \mathrm{~kg}$ de peso vivo do cordeiro. $\mathrm{Na}$ Tabela 4, o consumo de matéria seca e a conversão alimentar foram calculados especificamente para os cordeiros, uma vez que as ovelhas, já haviam sido retiradas do confinamento.

Observa-se que para todas as variáveis estudadas, tanto para o conjunto ovelha + cordeiro (Tabela 3) como para os cordeiros (Tabela 4), não foram encontradas diferenças $(\mathrm{P} \geq 0,05)$ entre tratamentos. Comparando o consumo de matéria seca em termos percentuais médios (\% PV), verifica-se que praticamente não houve diferença entre ovelhas + cordeiros e cordeiros $(2,34 \%$ x $2,41 \%)$. Isso se deve ao fato de que o peso do cordeiro acrescido ao da 
Tabela 2 - Médias e erros-padrões para consumo médio diário de matéria seca (CMS), de cordeiros do desmame aos 75 dias (50-75) e dos 75 aos 100 dias (75-100) de confinamento.

\begin{tabular}{|c|c|c|c|c|c|c|}
\hline \multirow[b]{3}{*}{ TRATAMENTO } & \multicolumn{6}{|c|}{ CMS } \\
\hline & \multicolumn{2}{|c|}{ (kg/an/dia) } & \multicolumn{2}{|c|}{$(\% \mathrm{PV})$} & \multicolumn{2}{|c|}{ (g/UTM) } \\
\hline & $50-75$ & $75-100$ & $50-75$ & $75-100$ & $50-75$ & $75-100$ \\
\hline Macho Inteiro & 0,48 & 0,68 & 2,20 & 2,65 & 47,41 & 59,79 \\
\hline Macho Castrado & 0,49 & 0,68 & 2,21 & 2,64 & 47,93 & 59,40 \\
\hline Fêmea & 0,44 & 0,63 & 2,11 & 2,56 & 45,22 & 56,92 \\
\hline Média & 0,47 & 0,66 & 2,17 & 2,61 & 46,85 & 58,71 \\
\hline Erro Padrão & 0,05 & 0,05 & 0,19 & 0,13 & 4,20 & 3,31 \\
\hline $\mathrm{F}$ & 0,26 & 0,32 & 0,08 & 0,15 & 0,12 & 0,22 \\
\hline $\mathrm{P}>\mathrm{F}$ & 0,78 & 0,73 & 0,93 & 0,86 & 0,89 & 0,80 \\
\hline $\mathrm{CV}(\%)$ & 23,82 & 19,78 & 21,68 & 12,33 & 22,02 & 13,82 \\
\hline
\end{tabular}

$(\mathrm{P} \geq 0,05)$.

\begin{tabular}{|c|c|c|c|c|}
\hline \multirow[b]{2}{*}{ TRATAMENTO } & \multicolumn{3}{|c|}{ CMS } & \multirow[t]{2}{*}{$\mathrm{CA}$} \\
\hline & (kg/an/dia) & $(\% \mathrm{PV})$ & (g/UTM) & \\
\hline Macho Inteiro & 1,42 & 2,28 & 56,10 & 4,61 \\
\hline Macho Castrado & 1,51 & 2,45 & 59,87 & 4,63 \\
\hline Fêmea & 1,44 & 2,28 & 56,34 & 4,56 \\
\hline Média & 1,46 & 2,34 & 57,44 & 4,60 \\
\hline Erro Padrão & 0,07 & 0,10 & 2,37 & 0,32 \\
\hline $\mathrm{F}$ & 0,39 & 0,97 & 0,79 & 0,01 \\
\hline$P>F$ & 0,69 & 0,40 & 0,47 & 0,99 \\
\hline $\mathrm{CV}(\%)$ & 12,81 & 10,49 & 10,14 & 17,23 \\
\hline
\end{tabular}

$(\mathrm{P} \geq 0,05)$.

ovelha, dilui o consumo em termos percentuais, principalmente no período inicial, onde a participação do cordeiro na ingestão de alimento sólido é muito pouca. Para o consumo em relação ao peso metabólico, observa-se que o conjunto (ovelha + cordeiro) apresentou um consumo médio de $57,44 \mathrm{~g} / \mathrm{UTM}$ e os cordeiros de 53,01g/UTM. A superioridade das ovelhas + cordeiros está relacionada ao maior consumo diário de alimento.

Os dados médios deste experimento para consumo diário de matéria seca dos cordeiros (Tabela 4) foram inferiores aos obtidos por PIRES et al. (1996), que obtiveram um consumo médio de 0,915kg/animal/dia, PILAR et al. (1994), que observaram um consumo médio diário de MS de $1093 \mathrm{~g}$
(Hampshire Down), 828g (Texel), 874g (Corriedale), 924g (Suffolk x Corriedale) e $869 \mathrm{~g}$ (Ile de France x Corriedale) e ARAÚJO (1996), que observou um consumo médio diário de MS de $733 \mathrm{~g}$ (Ideal), $853 \mathrm{~g}(1 / 2$ Texel $x 1 / 2$ Ideal $)$ e $887 \mathrm{~g}(3 / 4$ Texel $\mathrm{x} 1 / 4$ Ideal).

Os maiores consumos diários de matéria seca encontrados pelos autores citados acima, podem ser explicados pela menor idade dos cordeiros do presente trabalho, o que determina uma melhor eficiência de utilização de alimento e também um menor desenvolvimento do trato digestivo, o que resulta em consumo inferior. Em relação aos trabalhos desenvolvidos por ARAÚJO (1996) e PIRES et al. (1996), as diferenças encontradas podem também terem sido determinadas pela qualidade do alimento utilizado por esses autores na alimentação dos animais, pois utilizaram como volumoso, respectivamente, feno de azevém e feno de mi-

lheto.

Quando os valores de consumo de matéria seca foram expressos em percentual (\% PV) ou em g/UTM, observou-se que os resultados obtidos neste experimento, tanto para ovelha + cordeiro, como somente para os cordeiros, foram inferiores aos obtidos por PIRES et al. (1996), com 3,5\% e 80g, PILAR et al. (1994), com valores médios de 2,83\% e 67g e ARAÚJO (1996), que encontrou 3,14\% e $69 \mathrm{~g}$ (Ideal), 3,28\% e 74g ( $1 / 2$ Texel $+1 / 2$ Ideal) e $3,31 \%$ e $75 \mathrm{~g}(3 / 4$ Texel $+1 / 4$ Ideal $)$.

Com relação à conversão alimentar, observa-se que a mesma melhorou com a retirada da ovelha do confinamento. Isso ocorreu, porque no primeiro período, como a conversão alimentar foi calculada em função da quantidade de alimento consumida pelo conjunto ovelha + cordeiro, o consumo diário de alimento era muito grande em função da participação da ovelha, determinando uma pior conversão alimentar. Mesmo assim, verifica-se uma boa eficiência do conjunto, o que se deve ao ótimo ganho de peso dos cordeiros que se encontravam em uma fase de máximo potencial de crescimento.

Os resultados obtidos, neste trabalho, tanto para ovelhas + cordeiros como para os cordeiros, em relação à conversão alimentar, foram superiores aos obtidos por DEUZAMAR et al. (1994), que trabalhando com ovinos mestiços Santa Inês, obtiveram um valor médio 5,95:1. Foram superiores, também, aos resultados obtidos por PILAR et al. (1994), que obtiveram valores para conversão alimentar de 6,73 na raça Hampshire Down, 6,42 na raça Texel, 
Tabela 4 - Médias e erros-padrões para consumo médio diário de matéria seca (CMS) e conversão alimentar (CA) de cordeiros, dos 50 aos 100 dias de idade.

\begin{tabular}{lrrrr}
\hline & \multicolumn{5}{c}{ CMS } & CA \\
TRATAMENTO & (kg/an/dia) & $(\%$ PV $)$ & $(\mathrm{g} / \mathrm{UTM})$ \\
& & & & \\
\hline & & & & \\
Macho Inteiro & 0,58 & 2,43 & 53,69 & 3,76 \\
Macho Castrado & 0,58 & 2,45 & 54,19 & 4,30 \\
Fêmea & 0,54 & 2,34 & 51,14 & 4,55 \\
Média & 0,57 & 2,41 & 53,01 & 4,20 \\
Erro Padrão & 0,05 & 0,15 & 3,48 & 0,39 \\
F & 0,33 & 0,16 & 0,22 & 1,10 \\
P>F & 0,72 & 0,85 & 0,81 & 0,36 \\
CV $(\%)$ & 19,77 & 15,43 & 16,09 & 22,54 \\
& & & & \\
\hline
\end{tabular}

$(\mathrm{P} \geq 0,05)$.

Suffolk x Corriedale e 6,18 em animais cruza Ile de France x Corriedale. Cabe salientar que, em ambos os trabalhos citados acima, os valores foram obtidos com animais submetidos ao regime de confinamento e com uma maior idade que os do presente estudo.

Observa-se que os cordeiros machos, não castrados após o desmame, foram os que apresentaram os melhores índices de conversão alimentar (3,76:1), evidenciando uma possível melhora na eficiência de utilização de alimento por parte dos animais não castrados. Estes valores estão de acordo com FIELD (1971), que observou que cordeiros machos não castrados são 12 a $15 \%$ mais eficientes na conversão do alimento em ganho de peso vivo do que os castrados, e que a eficiência alimentar é incrementada com o aumento da taxa de ganho de peso.

\section{CONCLUSÕES}

O sistema de confinamento pode ser utilizado com eficiência na alimentação de cordeiros na fase de cria e terminação. Quanto ao desempenho, consumo e conversão alimentar, não há evidência de superioridade de machos não castrados em relação a machos castrados e fêmeas, quanto abatidos em idade muito jovem. Este fato mostra que as fêmeas podem ser utilizadas com eficiência para produção de carne ovina.

\section{REFERÊNCIAS BIBLIOGRÁFICAS}

ARAÚJO, J.R. Desempenho e características da carcaça de cordeiros de três grupos genéticos abatidos a mesma maturidade. Santa Maria: UFSM. 74 p. Dissertação (Mestrado em Zootecnia) - Universidade Federal de Santa Maria, 1996.
5,78 na raça Corriedale, 5,78 em animais cruzas AZZARINI, M. Produção de carne ovina. In: $1^{\mathrm{a}}$ JORNADA TÉCNICA DE PRODUÇÃO OVINA NO RIO GRANDE DO SUL, 1., Bagé. Anais... Bagé: EMBRAPA, 1979, p. 49-63.

CASTEllá, J.C. Quem disse que é inviável confinar? A Granja: Taxa de retorno melhor que a do boi, n. 580, p. 59$61,1997$.

CROUSE, J.D., PUSHOOM, J.R., FIELD, R.A. et. al. The effects of breed, sex, location and slaughter weight on lamb growth carcass composition and meat flavour. Journal of Animal Science. v. 53, p. 376-386, 1981.

DEUZAMAR, M.F.S., SILVA, D.S., BANDEIRA, D.A., $\boldsymbol{e}$ t al Efeito da substituição da proteína de soja por uréia na alimentação de ovinos confinados. In: XXXI REUNIÃO ANUAL DA SOCIEDADE BRASILEIRA DE ZOOTECNIA, 1994, Maringá. Anais... Maringá: Sociedade Brasileira de Zootecnia, 1994, p. 423.

FIELD, R.A. Effect of castration on meat quality and quantity. Journal of Animal Science, v. 32, n. 5, p. 849-858, 1971.

FIGUEIRÓ, P.R.P.; BENAVIDES, M.V. Produção de carne ovina. In: SIMPÓSIO SOBRE PRODUÇÃO ANIMAL, 7, 1990. Campinas, SP. Anais... Piracicaba. SP. FEALQ, p. 171$187,245 \mathrm{p}$.

JACOBS, J.A., FIELD, R.A., BOTKIN, M.P., et al. Effects of testosterone enanthate on lamb carcass composition and quality. Journal of Animal Science. v. 34, n. 1, p. 30, 1972.

JENKINS, T.G., FORD, J.J., KLINDT, J. Postweaning growth, feed efficiency and chemical composition of sheep as affected by prenatal and postnatal testosterone. Journal of Animal Science. v. 66, p. 1179-1185, 1988.

MOREIRA, N. Quem disse que é inviável confinar ? A Granja. n.580, p. 59-61, 1997.

NATIONAL RESEARCH COUNCIL (NRC). Nutrient Requirements of Sheep. 6 th ed. Washington: National Academy Press, 1985, 99 p.

PILAR, R.C., PIRES,C.C., RESTLE, J. et al. Desempenho em confinamento e componentes do peso vivo de diferentes genótipos de ovinos abatidos aos 12 meses de idade. Ciência Rural, Santa Maria, n. 3, p. 607-612, 1994.

PIRES, C.C., ROSA, G.T., GONÇALVES, J. et al. Desempenho e características da carcaça de cordeiros terminados em confinamento. In: XXXIII REUNIÃO ANUAL DA SOCIEDADE BRASILEIRA DE ZOOTECNIA. Fortaleza, Anais... Fortaleza: Sociedade Brasileira de Zootecnia, 1996.

SAS. Copyright 1990 by SAS Institut Inc. Cary, North Caroline, USA.

SENTS, A.E., WALTERS, L.E., WHITEMAN, J.V. Performance and carcass characteristics of ram lambs slaughtered at different weights. Journal of Animal Science. v. 55, n. 6, p. 1360-1368, 1982.

SIQUEIRA, E.R., AMARANTE, A.F.T., FERNANDES, S. Estudo comparativo da recria de cordeiros em confinamento e pastagem. Revista Veterinária e Zootecnia. v. 5, p. 17-28, 1993. 